\title{
Cherenkov Radiation from the Quantum Vacuum
}

\author{
Alexander J. Macleod, Adam Noble, ${ }^{*}$ and Dino A. Jaroszynski ${ }^{\dagger}$ \\ SUPA Department of Physics, University of Strathclyde, Glasgow G4 ONG, United Kingdom
}

(Received 17 September 2018; revised manuscript received 1 March 2019; published 24 April 2019)

\begin{abstract}
A charged particle moving through a medium emits Cherenkov radiation when its velocity exceeds the phase velocity of light in that medium. Under the influence of a strong electromagnetic field, quantum fluctuations can become polarized, imbuing the vacuum with an effective anisotropic refractive index and allowing the possibility of Cherenkov radiation from the quantum vacuum. We analyze the properties of this vacuum Cherenkov radiation in strong laser pulses and the magnetic field around a pulsar, finding regimes in which it is the dominant radiation mechanism. This radiation process may be relevant to the excess signals of high energy photons in astrophysical observations.
\end{abstract}

DOI: 10.1103/PhysRevLett.122.161601

Quantum electrodynamics (QED) is one of the most successful and well tested theories in physics. An early prediction of QED is the presence of virtual particleantiparticle pairs which fluctuate in and out of existence in the quantum vacuum. It has been known since the seminal work of Euler and Heisenberg [1] (see also [2]) that a strong electromagnetic field can polarize these vacuum fluctuations. This, in turn, can mediate an indirect interaction between a probe photon and the strong field such that the photon propagates as it would in a dielectric medium (for extensive reviews, see [3-5] and references therein). The Euler-Heisenberg theory is the result of integrating out the fermion degrees of freedom in the QED path integral, producing a nonlinear effective theory in which the photon interacts directly with the strong field. Other nonlinear theories of electrodynamics have been proposed, most notably the Born-Infeld theory [6], which, in its original form, was an attempt to resolve the electron self-energy problem before the advent of QED. More recently, it has found a resurgence of interest due to its emergence in the low energy limit of some string theories $[7,8]$.

It is well known that a charged particle moving through a material medium can emit Cherenkov radiation $[9,10]$. The first theoretical work to explain these results was presented by Frank and Tamm [11] (though earlier work by Heaviside [12] and Sommerfeld [13] considered similar effects). This effect occurs because, in a medium with refractive index $n$, the phase velocity of light is reduced, $v_{p}=c / n$, so a particle traveling through the medium with velocity $V>v_{p}$ will outrun any electromagnetic waves it emits. This can

Published by the American Physical Society under the terms of the Creative Commons Attribution 4.0 International license. Further distribution of this work must maintain attribution to the author(s) and the published article's title, journal citation, and DOI. Funded by SCOAP. lead to the emission of radiation due to the buildup of wave fronts propagating from the particle, producing the well known "Cherenkov cone" of radiation behind the particle.

Since vacuum fluctuations can also reduce the phase velocity of light (see, for example, [14]), the same argument implies that high-energy particles traveling through strong electromagnetic fields should emit Cherenkov radiation, in addition to the usual synchrotron radiation caused by acceleration in the field. First steps towards analyzing this effect were taken by Erber [15], who used the principles of QED to obtain semiquantitative predictions for the radiation emitted by an electron in a strong magnetic field. This was followed by Ritus [16], who derived the analogous process for an electron in constant crossed fields from the effective photon mass. Subsequently, Dremin [17] made more quantitative estimates for the Cherenkov radiation produced by particles crossing a laser pulse, while Marklund et al. [18] explored the possibility of Cherenkov radiation from a particle in a photon gas.

In this Letter, we provide a unified description of vacuum Cherenkov radiation in nonlinear electrodynamics, applicable to arbitrary field configurations. To illustrate the approach, we analyze the effect in the context of both upcoming laser facilities (e.g., the Extreme Light Infrastructure (ELI) [19]) and astrophysical sources of strong fields. In the latter, we find regimes in which the Cherenkov radiation dominates over other radiation processes, highlighting a new and, as yet, unexplored mechanism for generating gamma rays, which we suggest should be further investigated in the context of the observed excess signals of astrophysical high energy photons [20-23].

Lorentz invariance of the vacuum requires nonlinear theories of electrodynamics to be constructed from Lagrangians, $\mathcal{L}(X, Y)$, depending only on the two electromagnetic invariants, $X=-\frac{1}{4} F^{\mu \nu} F_{\mu \nu}$ and $Y=-\frac{1}{4} \tilde{F}^{\mu \nu} F_{\mu \nu}$, where $F^{\mu \nu}$ and $\tilde{F}^{\mu \nu}$ are the electromagnetic field and its dual, and repeated indices imply summation. Given the 
success of Maxwell's theory, we consider only leading order corrections, i.e., Lagrangians of the form

$$
\mathcal{L}=X+\lambda_{+} X^{2}+\lambda_{-} Y^{2},
$$

where the constants $\lambda_{ \pm}$determine the specific theory. For Euler-Heisenberg,

$$
\frac{1}{4} \lambda_{+}=\frac{1}{7} \lambda_{-}=\frac{\alpha}{90 \pi} \frac{1}{E_{S}^{2}},
$$

where $\alpha \simeq 1 / 137$ is the fine-structure constant and $E_{S}=$ $m_{e}^{2} / e \simeq 1.3 \times 10^{18} \mathrm{~V} / \mathrm{m}$ is the Schwinger field [2] (we work throughout in units where $c=\hbar=1$ ). In the BornInfeld theory, the (unknown) constants coincide, $\lambda_{+}=\lambda_{-}$ [6]. Although our results are readily extendible to more general Lagrangians, (1), (2) remains a good approximation for field strengths approaching $E_{S}$ and so is sufficient for our purposes.

The field equations following from (1) are $\partial_{\mu} \tilde{F}^{\mu \nu}=0$ and $\partial_{\mu} H^{\mu \nu}=0$, where the excitation tensor $H^{\mu \nu}=$ $\left(1+2 \lambda_{+} X\right) F^{\mu \nu}+2 \lambda_{-} Y \tilde{F}^{\mu \nu}$. Taking $F^{\mu \nu}$ to be the sum of a strong, slowly varying background $\mathcal{F}^{\mu \nu}$ and a weaker radiation field $f^{\mu \nu}$, and linearizing in the latter, yields

$$
\partial_{\mu} \tilde{f}^{\mu \nu}=0, \quad \partial_{\mu}\left(\chi^{\mu \nu \alpha \beta} f_{\alpha \beta}\right)=0,
$$

with the constitutive tensor

$$
\begin{aligned}
\chi^{\mu \nu \alpha \beta}= & \left(1+2 \lambda_{+} \mathcal{X}\right)\left(g^{\mu \alpha} g^{\nu \beta}-g^{\mu \beta} g^{\nu \alpha}\right) \\
& -2 \lambda_{+} \mathcal{F}^{\mu \nu} \mathcal{F}^{\alpha \beta}-2 \lambda_{-} \tilde{\mathcal{F}}^{\mu \nu} \tilde{\mathcal{F}}^{\alpha \beta} .
\end{aligned}
$$

$g^{\mu \nu}$ is the metric tensor, and we define $\mathcal{X}=-\frac{1}{4} \mathcal{F}^{\mu \nu} \mathcal{F}_{\mu \nu}$ and similarly for $\mathcal{Y}$.

The system (3), (4) has been well studied (e.g., [24,25]). Neglecting derivatives of the background [26], and defining the phase $\varphi=k_{\mu} x^{\mu}$, the radiation field can be expressed as $f_{\mu \nu}=\left(k_{\mu} a_{\nu}-k_{\nu} a_{\mu}\right) e^{i \varphi}$, where the polarization $a_{\mu}$ and wave vector $k_{\mu}$ are determined algebraically from

$$
\chi^{\mu \nu \alpha \beta} k_{\nu} k_{\beta} a_{\alpha}=0 .
$$

This has solutions $a_{+}^{\mu}=\mathcal{F}^{\mu}{ }_{\nu} k_{+}^{\nu}, a_{-}^{\mu}=\tilde{\mathcal{F}}^{\mu}{ }_{\nu} k_{-}^{\nu}$ [24], with the wave vectors obeying the dispersion relations $k_{ \pm}^{2} \simeq 2 \lambda_{ \pm} \mathcal{F}_{\lambda \mu} \mathcal{F}_{\nu}^{\lambda} k_{ \pm}^{\mu} k_{ \pm}^{\nu}$, where only the leading order behavior in $\lambda_{ \pm}$has been included. Evidently, for $\lambda_{+} \neq \lambda_{-}$, we have birefringence. Using $k^{2}=\left(\omega^{2}-|\mathbf{k}|^{2}\right)=$ $\left(v_{p}^{2}-1\right)|\mathbf{k}|^{2}$, the dispersion relations yield the phase velocity $v_{p}$

$$
v_{p \pm}^{2} \simeq 1+2 \lambda_{ \pm} \mathcal{F}_{\lambda \mu} \mathcal{F}^{\lambda}{ }_{\nu} \hat{k}_{ \pm}^{\mu} \hat{k}_{ \pm}^{\nu},
$$

where $\hat{k}_{ \pm}^{\mu}=k_{ \pm}^{\mu} /\left|\mathbf{k}_{ \pm}\right|$is the direction 4-vector of the radiation. For Euler-Heisenberg, the last term in (6) encodes the effect of the photon mass operator in QED $[15,16]$.

To interpret these solutions as Cherenkov radiation, we must relate them to the source of the radiation-i.e., the charged particle. The analogous problem in a material medium has been well studied [11] and leads to the wellknown expressions for the emission angle $\theta_{C}$ relative to the particle's velocity and the power radiated per unit frequency $d P / d \omega$

$$
\cos \theta_{C}=\frac{v_{p}}{|\boldsymbol{\beta}|}, \quad \frac{d P}{d \omega}=\frac{e^{2}}{4 \pi} \omega \sin ^{2} \theta_{C}
$$

These are calculated for Cherenkov radiation in a homogeneous, isotropic medium (ICR). Each of the expressions (7) are relatively simple in their structure, and it can be clearly seen that the key parameters are the phase velocity $v_{p}$, and the particle velocity $\boldsymbol{\beta}$. The definition of the Cherenkov angle ensures that no Cherenkov radiation is observed for $\beta<v_{p},(\beta \equiv|\boldsymbol{\beta}|)$.

The generalization of the Cherenkov angle to nonlinear electrodynamics is straightforward: it retains the form given in (7), but the anisotropy of the background field implies the phase velocity itself depends on the direction of emission, $v_{p}=v_{p}(\hat{k})$. This can be accounted for using (6)

$$
\cos ^{2} \theta_{C}^{ \pm}=\frac{1}{\beta^{2}}\left(1+2 \lambda_{ \pm} \mathcal{F}_{\lambda \mu} \mathcal{F}^{\lambda}{ }_{\nu} \hat{k}_{ \pm}^{\mu} \hat{k}_{ \pm}^{\nu}\right)
$$

which is valid in any (slowly varying) background field. Note that, in theories exhibiting birefringence, we have two Cherenkov cones, corresponding to the different phase velocities of the two polarizations.

It is clear that the Cherenkov power formula in (7) cannot be adopted directly into the nonlinear theories as, in general, $\theta_{C}^{ \pm}$depends on the azimuthal angle, and we must, instead, determine the differential power emitted per unit frequency per unit azimuthal angle, $d^{2} P / d \omega d \phi$. We follow the approach taken by Altschul to describe Cherenkov radiation in Lorentz-violating vacua [27]. Although the physical basis of such theories is the reverse of nonlinear electrodynamics (where Lorentz invariance is strictly preserved), the linearization treats the background field as an external structure, and (3) is formally equivalent to $C P T$ even Lorentz-violating electrodynamics. The key observation is that Cherenkov modes corresponding to different wave vectors $k^{\mu}$ propagate independently and, hence, behave as waves propagating in an isotropic medium with scalar refractive index $n=1 / v_{p}(\hat{k})$. ICR is linearly polarized in the plane $(\hat{\boldsymbol{\beta}}, \hat{\mathbf{k}})$, and orthogonal to $\hat{\mathbf{k}}$, i.e., in the direction $\hat{\boldsymbol{\epsilon}}_{\boldsymbol{0}}=\left(\hat{\boldsymbol{\beta}}-\cos \theta_{C} \hat{\mathbf{k}}\right) / \sin \theta_{C}$ (throughout, spatial vectors with carets are unit normalized). In the nonlinear case, there are two independent polarization modes, $a_{+}^{\mu}$ and $a_{-}^{\mu}$, the spatial parts of which do not, in general, coincide with $\hat{\boldsymbol{\epsilon}}_{\mathbf{0}}$. As such, only the projection of ICR along these directions will propagate 


$$
\frac{d^{2} P_{ \pm}}{d \omega d \phi}=\frac{e^{2}}{8 \pi^{2}}\left|\hat{\boldsymbol{\epsilon}}_{\mathbf{0}} \cdot \hat{\boldsymbol{\epsilon}}_{ \pm}\right|^{2} \omega \sin ^{2} \theta_{C}^{ \pm}
$$

Here, $\hat{\boldsymbol{\epsilon}}_{ \pm}$are the (unit normalized) spatial components of the polarization modes $a_{ \pm}^{\mu}$ (see Supplemental Material [28] for details). The derivation of (9) treats the particle's orbit as rectilinear. Therefore, it is valid only for wavelengths that the particle can emit while turning a negligible angle. See Supplemental Material [28] for a demonstration that this includes almost all the radiation in the examples below.

As can be seen from (7) and (9), the Cherenkov spectrum has an explicit linear dependence on the frequency $\omega$. This means that the spectrum appears to diverge at high frequencies. In a material medium, dispersive effects give $\theta_{C}$ an $\omega$ dependence, so that, at high frequencies, Cherenkov radiation is suppressed. In nonlinear electrodynamics, this is not the case, and we must assume a cutoff frequency will arise from physics not captured in $\mathcal{L}(X, Y)$ (see [29] for an analogous discussion in the context of Lorentz-violating electrodynamics). In the case of QED, for example, the Euler-Heisenberg Lagrangian must be supplemented by higher derivative terms at very high frequencies [3]. We could simply impose a cutoff directly on the frequency $\omega$. However, since frequency is not a Lorentz invariant, this would not be a physically meaningful condition. Instead, we assume (9) is valid for photons with a small quantum nonlinearity parameter [16]

$$
\chi_{\gamma}=\frac{|e|}{m_{e}^{3}} \sqrt{-\mathcal{F}^{\mu \lambda} \mathcal{F}^{\nu}{ }_{\lambda} k_{\mu} k_{\nu}} \lesssim 1,
$$

which can be solved for the maximum frequency, $\omega_{\max }$. This is not strictly a cutoff, and Cherenkov radiation may still occur for higher frequencies, but our results may not be reliable above $\omega_{\max }$.

In principle, the Lorentz factor $\gamma$ should reduce as the particle loses energy to radiation. This could be accounted for by introducing a damping force $\mathbf{F}_{d}=-\mathcal{P}_{C} \boldsymbol{\beta}$, where $\mathcal{P}_{C}$ is the integral of (9), and solving simultaneously for the motion of the particle and the radiation. In practice, however, this is generally unnecessary, as, for $\gamma \gg 1 / \sqrt{1-v_{p}^{2}}$, we can set $\beta=1$ in (8), (9).

With these considerations, we now have all the ingredients necessary to determine the Cherenkov radiation emitted by a particle moving in any given field configuration. To demonstrate more concretely the vacuum Cherenkov effect, we consider two examples of field configurations: a constant crossed field (representing a laser pulse) and a constant magnetic field (representing the field around a pulsar).

Advances in laser technology have begun to provide a platform to study strong field effects experimentally, for example, the recent results concerning radiation reaction [30,31]. Many results pertaining to strong field physics locally approximate the laser beam as a constant crossed field. We will consider the background field $\mathcal{F}^{\mu \nu}$ to represent a constant crossed field (i.e., $\mathcal{X}=\mathcal{Y}=0$ ) of strength $E$, with Poynting vector in the $\hat{\mathbf{z}}$ direction. We consider the electron to be counterpropagating with respect to the Poynting vector, as, in this configuration, the energy transfer between background and electron will be greatest, leading to the strongest effect. With the set up described, the phase velocity can be determined via (6), which leads to the simple expression for the Cherenkov angles

$$
\cos ^{2} \theta_{C}^{ \pm}=\frac{1}{\left[\beta^{2}+2(1+\beta)^{2} \lambda_{ \pm} E^{2}\right]} .
$$

In this case, the Cherenkov angles are independent of the azimuthal angle $\phi$ and yield the Cherenkov condition $\left(\gamma+\sqrt{\gamma^{2}-1}\right)^{2} E^{2}>1 / 2 \lambda_{ \pm}$. Cherenkov radiation will occur whenever this condition is satisfied; however, to be observable, it must be non-negligible in comparison with the emission of synchrotron radiation by electrons oscillating in the field. The synchrotron spectrum is [32]

$$
\frac{d P_{\mathrm{synch}}}{d \omega}=\frac{\sqrt{3}}{\pi} \frac{e^{3} E}{m_{e} c^{3}} \frac{\omega}{\omega_{c}} \int_{\omega / \omega_{c}}^{\infty} d x K_{5 / 3}(x),
$$

where $K_{\nu}(x)$ is the order $\nu$ modified Bessel function of the second kind and $\omega_{c}=3\left(e E / m_{e} c\right) \gamma^{2}$. To compare the two radiation processes, we integrate the Cherenkov spectrum with respect to the azimuthal angle and consider the total power per unit frequency

$$
\frac{d P_{\text {Cher }}}{d \omega}=\int_{0}^{2 \pi} d \phi\left(\frac{d^{2} P_{+}}{d \omega d \phi}+\frac{d^{2} P_{-}}{d \omega d \phi}\right),
$$

where the contribution from each individual mode is determined by (9).

Future laser facilities such as ELI [19] are expected to reach field strengths on the order of $E \sim E_{S} \times 10^{-3}$, with access to electrons up to $\gamma \sim 10^{5}(\simeq 50 \mathrm{GeV})$. Thus, we consider this parameter regime in comparing the spectra from Cherenkov and synchrotron radiation in a constant crossed field. We also specialize to the Euler-Heisenberg Lagrangian (2), as this arguably represents the best motivated nonlinear extension to Maxwell electrodynamics. Figure 1 shows the calculated power per unit frequency due to each of the radiation processes as a function of the emitted photon energy $\hbar \omega$. The black dashed line represents the cutoff found from (10), $\hbar \omega_{\max } \sim\left(m_{e}^{3} c^{5}\right) /$ $(2 e \hbar E) \simeq 0.25 \mathrm{GeV}$. Below this limit, synchrotron radiation is always the dominant process. Thus, observing the Cherenkov effect appears unlikely for even future laser facilities. This is primarily due to the limitation on the ability to produce high energy electrons in the lab. For $\gamma \gg 1$, the Cherenkov spectrum becomes proportional to $E^{2}$, to leading order, and so, for a fixed field strength, 


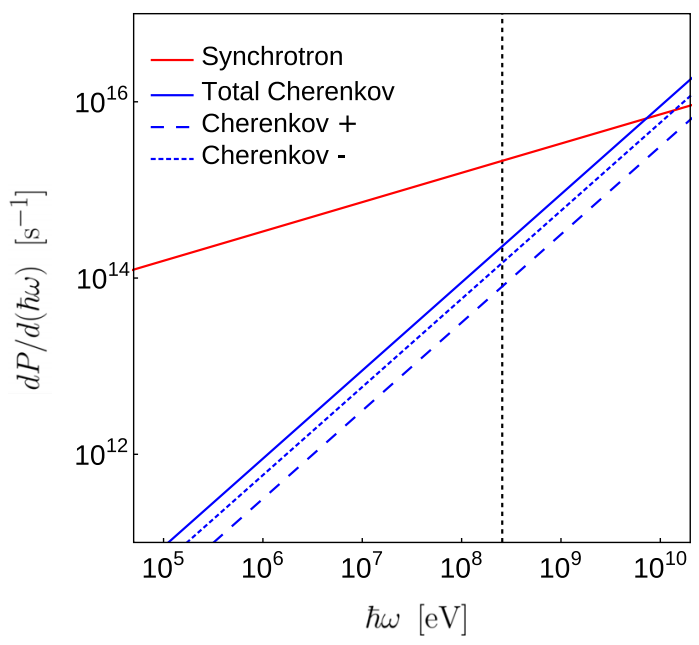

FIG. 1. Radiated power from the interaction of an electron with $\gamma=10^{5}$ and a crossed field with field strength $E=E_{S} \times 10^{-3}$, due to: Synchrotron radiation (red line); total Cherenkov radiation (blue, solid line); Cherenkov + mode (blue, dashed line); Cherenkov - mode (blue, dotted line). The cutoff energy (black, dashed line) is $\hbar \omega_{\max } \simeq 0.25 \mathrm{GeV}$.

increasing the energy of the particles has very little effect on the Cherenkov spectrum. Conversely, the synchrotron spectrum becomes increasingly suppressed as $\gamma$ increases for fixed $E$. For the field strength considered here, an electron Lorentz factor $\gamma \sim 2.5 \times 10^{6}$, corresponding to an energy of $1.3 \mathrm{TeV}$, would be required to have the contributions from Cherenkov and synchrotron processes approximately equal at the cutoff. There is also the concern that to reach these high field strengths in a real experiment, strong focusing techniques are needed to compress the laser pulse, and this brings in a significant range of other effects which would act to drown out the Cherenkov signal, or deplete the electron energy sufficiently that, by the time it reaches the peak intensity of the pulse, its energy has fallen below the Cherenkov threshold [33]. It might be hoped that protons offer a viable alternative, since their mass greatly suppresses synchrotron radiation. However, the Cherenkov threshold corresponds to a proton energy of $33 \mathrm{TeV}$, well beyond what can currently be produced. Therefore, the possibility of observing Cherenkov radiation in this context seems bleak.

Since the main obstacle to observing Cherenkov radiation is the availability of high energy particles, it is natural to turn our attention to astrophysics, where the only limit on the particle energy is the so-called Greisen-ZatsepinKuzmin limit, $\gamma \lesssim 10^{11}[34,35]$. Astrophysical objects such as pulsars have also been observed to generate magnetic fields up to and exceeding the Schwinger magnetic field $B_{S}=E_{S} / c \simeq 4.4 \times 10^{9} \mathrm{~T}$. This makes such a scenario ideal for the study of nonlinear vacuum Cherenkov radiation. As such, we consider a constant magnetic field of strength $B$. We take the particle's velocity perpendicular to the field, since any parallel component of $\boldsymbol{\beta}$ can be removed by a Lorentz transformation which does not alter the form of the background field. Taking the $\hat{\mathbf{z}}$ axis along the particle's velocity, the polar angle of the emitted radiation and the Cherenkov angle coincide, $\theta=\theta_{C}$. This immediately gives an azimuthal dependence to the Cherenkov angle

$$
\cos ^{2} \theta_{C}^{ \pm}=\frac{1-2 \lambda_{ \pm} B^{2} \cos ^{2} \phi}{\beta^{2}+2 \lambda_{ \pm} B^{2} \sin ^{2} \phi}
$$

which gives the Cherenkov condition, $\gamma^{2} B^{2}>1 / 2 \lambda_{ \pm}$.

Again, we need to compare the Cherenkov and synchrotron spectra. In the case of the magnetic field, we use (12), with the substitution $E \rightarrow B c / 2$ (the factor 2 arises because the constant crossed field has both magnetic and electric components, essentially doubling the contribution). We are considering high energy cosmic rays, which are predominantly protons, so we consider the two radiation processes for these [36]. This amounts to changing $m_{e} \rightarrow m_{p}$ in (12). However, factors of $m_{e}$ appearing in the Cherenkov spectrum (through the parameters $\lambda_{ \pm}$) and the cutoff are not changed: the nonlinear terms in the Lagrangian (1), (2) and the mass scale in the cutoff (10) are determined by electron-positron fluctuations in the vacuum. The total power radiated per unit frequency is again determined by (13), with (9).

For radiation from protons, we also need to compare (13) with the radiation of pions, which, subsequently, decay into photons. The spectrum for such radiation is given by [37]

$$
\frac{d P_{\pi}}{d \omega}=\frac{g^{2}}{\sqrt{3} \pi c} \gamma^{-2} \omega \int_{y}^{\infty} d x K_{1 / 3}(x)
$$

where $\quad y=\frac{2}{3}\left(\hbar \omega / m_{e}\right)\left(m_{p} / m_{e}\right)\left(B_{S} / B\right) \gamma^{-2}[1+(\hbar \omega /$ $\left.\left.\gamma m_{\pi}\right)^{2}\right]^{3 / 2}, m_{\pi}$ is the pion mass, and $g^{2} \simeq 14 \hbar c$ is the pion-proton coupling strength.

The strongest magnetic fields observed are those produced by rapidly rotating pulsars. These objects have characteristic attributes of mass and radius, which, with rotational period, determine the typical field strengths produced. There are two broad classes of pulsar, those with a relatively longer rotational period which have magnetic field strengths $B \sim 10^{8} \mathrm{~T}$, and rapidly rotating "millisecond pulsars" which have typical field strengths $B \sim 10^{4} \mathrm{~T}$ [38]. The cutoff energy found through (10) is $\hbar \omega_{\max } \sim\left(m_{e}^{3} c^{4}\right) /(e \hbar B)$. This corresponds to $22.5 \mathrm{MeV}$ for $B=10^{8} \mathrm{~T}$, or $225 \mathrm{GeV}$ for $B=10^{4} \mathrm{~T}$. Since we are interested in high energy gamma rays, we illustrate the results for millisecond pulsars.

Figure 2 shows the spectra for Cherenkov, synchrotron, and pion radiation for a proton moving perpendicularly to a magnetic field $B=10^{4} \mathrm{~T}$, for $\gamma=5 \times 10^{7}$ (just above the Cherenkov threshold) and $\gamma=5 \times 10^{9}$. For clarity, we include only the total Cherenkov contribution. For $\gamma=5 \times 10^{7}$, the Cherenkov radiation exceeds synchrotron 


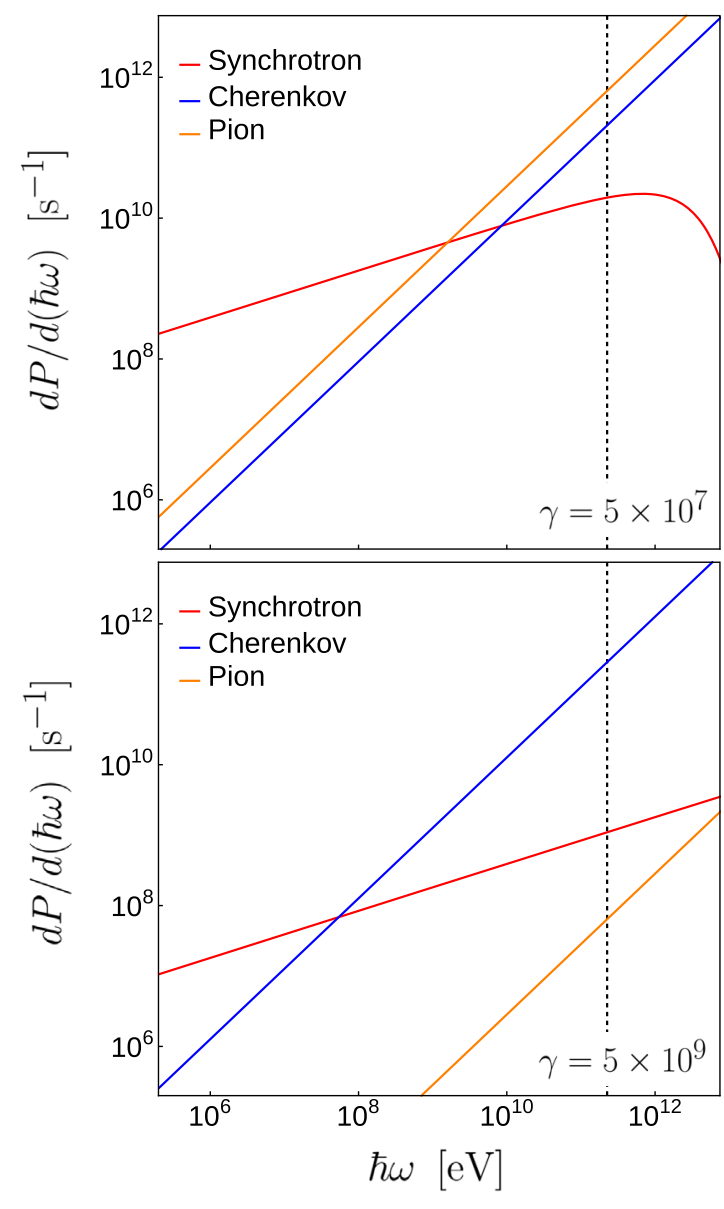

FIG. 2. Power radiated via synchrotron, pion, and Cherenkov emission, by protons in a magnetic field $B=10^{4} \mathrm{~T}$, with Lorentz factor $\gamma=5 \times 10^{7}$ (upper panel) and $\gamma=5 \times 10^{9}$ (lower panel). The cutoff energy is $\hbar \omega_{\max } \simeq 225 \mathrm{GeV}$.

emission for photon energies above $8.5 \mathrm{GeV}$ but remains below the pion emission up to $\hbar \omega_{\max }$. For $\gamma=5 \times 10^{9}$, however, Cherenkov radiation is, by far, the dominant emission channel for photon energies from $54 \mathrm{MeV}$ up to the cutoff. So for the highest energy proton cosmic rays, the highest energy radiation is completely dominated by the Cherenkov process.

There is currently a debate within the astrophysics community concerning the origin of observed excesses of high energy photons found in recent data. For example, observations of intense gamma rays from the Galactic Center [20] have prompted a range of possible explanations, such as dark matter annihilation [22] and unresolved pulsar sources [23]. The Cherenkov process detailed in this Letter provides a new, and so far unexplored, gamma-ray production mechanism, which we believe warrants further study in this context.

To summarize, in this Letter, we have provided a comprehensive, quantitative study of the Cherenkov effect in nonlinear theories of vacuum electrodynamics. This effect—expected due to the reduced phase velocity of light predicted by these theories in regions of strong fields-may provide an alternate radiation mechanism for very high energy particles. We considered two examples of background field with relevance to future experimental or observational campaigns, and determined the possibility of observing Cherenkov radiation in each case. When the background field is a constant crossed field (approximating a laser pulse), the availability of high energy particles appears to put observation of Cherenkov radiation out of reach. In contrast, astrophysics provides environments in which the vacuum Cherenkov effect may be observed due to the presence of very high energy cosmic rays and strong magnetic fields. We have demonstrated regimes in which radiation due to the nonlinear Cherenkov effect dominates over radiation produced through synchrotron and pion emission, generating very high energy photons. A notable excess of gamma rays with energies in the $\mathrm{GeV}-\mathrm{TeV}$ range has been observed in various astrophysical contexts, and the vacuum Cherenkov process could provide an alternate explanation for their origin, not previously considered in the literature.

We would like to thank other members of the ALPHA-X Collaboration for useful discussions. This work was supported by the UK EPSRC (Grant No. EP/N028694/1), the European Union H2020 Research and Innovation Programme LASERLAB EUROPE (654148) and a University of Strathclyde Doctoral Training Programme Studentship. All of the results can be fully reproduced using the methods described in the Letter.

*adam.noble@strath.ac.uk

d.a.jaroszynski@strath.ac.uk

*Present address: Centre for Mathematical Sciences, University of Plymouth, PL4 8AA, United Kingdom.

[1] W. Heisenberg and H. Euler, Z. Phys. 98, 714 (1936).

[2] J. Schwinger, Phys. Rev. 82, 664 (1951).

[3] M. Marklund and P. K. Shukla, Rev. Mod. Phys. 78, 591 (2006).

[4] A. Di Piazza, C. Müller, K. Z. Hatsagortsyan, and C. H. Keitel, Rev. Mod. Phys. 84, 1177 (2012).

[5] R. Battesti and C. Rizzo, Rep. Prog. Phys. 76, 016401 (2013).

[6] M. Born and L. Infeld, Proc. R. Soc. Ser. A 144, 425 (1934).

[7] E. Fradkin and A. Tseytlin, Phys. Lett. B 163, 123 (1985).

[8] F. Abalos, F. Carrasco, E. Goulart, and O. Reula, Phys. Rev. D 92, 084024 (2015)

[9] P. A. Cherenkov, C. R. Ac. Sci. U.S.S.R. 8, 451 (1934).

[10] S. Vavilov, C. R. Ac. Sci. U.S.S.R. 8, 457 (1934).

[11] I. Tamm and I. Frank, C. R. Ac. Sci. U.S.S.R. 14, 109 (1937).

[12] O. Heaviside, The Electrician 22, 147 (1888).

[13] A. Sommerfeld, Knkl. Acad. Wetensch. 7, 345 (1904).

[14] S. P. Flood and D. A. Burton, Europhys. Lett. 100, 60005 (2012).

[15] T. Erber, Rev. Mod. Phys. 38, 626 (1966).

[16] V. I. Ritus, J. Sov. Laser Res. 6, 497 (1985). 
[17] I. M. Dremin, JETP Lett. 76, 151 (2002).

[18] M. Marklund, G. Brodin, L. Stenflo, and P. K. Shukla, New J. Phys. 7, 70 (2005).

[19] Extreme Light Infrastructure, http://www.eli-np.ro/.

[20] M. Ackermann et al. (Fermi-LAT Collaboration), Astrophys. J. 840, 43 (2017).

[21] A. A. Abdo et al., Astrophys. J. Lett. 664, L91 (2007).

[22] D. Hooper and T. Linden, Phys. Rev. D 84, 123005 (2011).

[23] C. Gordon and O. Macías, Phys. Rev. D 88, 083521 (2013).

[24] Z. Bialynicka-Birula and I. Bialynicki-Birula, Phys. Rev. D 2, 2341 (1970).

[25] Y. N. Obukhov and G. F. Rubilar, Phys. Rev. D 66, 024042 (2002).

[26] This restricts our results to backgrounds that vary slowly on the scale of the radiation. However, since we are primarily interested in radiation of extremely short wavelengths, in practice, this includes all backgrounds one might want to consider.

[27] B. Altschul, Phys. Rev. D 75, 105003 (2007).
[28] See Supplemental Material at http://link.aps.org/ supplemental/10.1103/PhysRevLett.122.161601 for the relation between $\hat{\boldsymbol{\epsilon}}_{ \pm}$and $a_{ \pm}^{\mu}$.

[29] B. Altschul, Phys. Rev. Lett. 98, 041603 (2007).

[30] J. M. Cole et al., Phys. Rev. X 8, 011020 (2018).

[31] K. Poder et al., Phys. Rev. X 8, 031004 (2018).

[32] J. Schwinger, Phys. Rev. 75, 1912 (1949).

[33] Y. Kravets, A. Noble, and D. A. Jaroszynski, Phys. Rev. E 88, 011201(R) (2013).

[34] K. Greisen, Phys. Rev. Lett. 16, 748 (1966).

[35] G. T. Zatsepin and V. A. Kuz'min, JETP Lett. 4, 78 (1966).

[36] Very high energy electrons also emit Cherenkov radiation in the pulsar field. However, due to the greater rate of synchrotron emission in this case, the window in which Cherenkov radiation is the dominant effect is far narrower.

[37] V. L. Ginzburg and G. F. Zharkov, Sov. Phys. JETP 20, 1525 (1965).

[38] F. Camilo, S. E. Thorsett, and S. R. Kulkarni, Astrophys. J. 421, L15 (1994). 